\title{
NSLS X-19A BEAMLINE PERFORMANCE FOR X-RAY ABSORPTION MEASUREMENTS *
}

\author{
C.Y. YANG ** and J.E. PENNER-HAHN \\ Department of Chemistry, Unversity of Michigan, Ann Arbor, MI 48109, USA
}

\author{
P.M. STEFAN \\ National Synchrotron Light Source, Brookhaven National Laboratory, Upton, NY 11973, USA
}

\begin{abstract}
Characterization of the X-19 beamlıne at the National Synchrotron Light Source (NSLS) is described. The beamline is designed for high-resolution X-ray absorption spectroscopy over a wide energy range. All of the beamline optical components are compatible with ultrahigh vacuum (UHV) operation. This permits measurements to be made in a windowless mode, thereby facilitating lower energy $(<3 \mathrm{keV})$ studies. To upgrade the beamline performance, several possible improvements in instrumentation and practice are discussed to increase photon statistics with an optimum energy resolution while decreasing the harmonic contamination and noise level. A special effort has been made to improve the stability and UHV compatibility of the monochromator system. Inıtial X-ray absorption results demonstrate the capabilities of this beamlıne for X-ray absorption studıes of low- $Z$ elements (e.g., S) in highly dilute systems. The future use of this beamlıne for carrying out various $\mathrm{X}$-ray absorption experiments is presented
\end{abstract}

\section{Introduction}

Over the past decade, experimental and theoretical advances have made X-ray absorption spectroscopy (XAS) an important quantitative tool for probing the atomic structure, as well as the electronic and vibrational properties, of a wide range of materials. With the recent availability of strong and stable synchrotron radiation (SR) sources, the quality of the XAS data has dramatically improved so that complex features and weak signals of higher shells (within $\sim 5 \AA$ ) of the absorbing atom can be resolved in very dilute or disordered samples, which pose difficultues for other traditional techniques. Many of the available X-ray beamlines at SR sources were initially constructed to serve mult1ple purposes, and their design parameters are therefore a compromise between the different types of experimental needs. Using these "standard" XAS lines, it is often quite difficult to measure data for very dilute samples with high energy resolution in the low-energy X-ray region. (We refer to energies below $3 \mathrm{keV}$ as low energies.) For this reason, an effort has been made to upgrade the X-19A beamline at the NSLS. This beamline is dedicated to XAS measurements over a wide energy range. Particular emphasis has been placed on the low-

* Support for this research was provided by the Office of the Vice President for Research, University of Michıgan, and the U.S. Department of Energy under contract No. DEAC02-76CH0016.

** Also at Materials Science Division, Brookhaven National Laboratory, Upton, NY 11973, USA. energy range down to the $\mathrm{P}(2.15 \mathrm{keV})$ and $\mathrm{S}(2.47 \mathrm{keV})$ $\mathrm{K}$-absorption edges.

\section{Beamline setup}

At the X-19A beamlıne, X-rays are transported from the bending magnet source to the measuring apparatus through the system shown in fig. 1. The beamline accepts $3 \mathrm{mrad}$ of horizontal divergence from the source. Many of the primary components, such as the front end, the mirror box, the bender, the photon shutter, and the hutch, are based on standard NSLS designs. The first element of the beamline is the white-beam slit. This slit is used to limit the vertical divergence, to exclude scattered radiation, and to control the heat load on the first monochromator crystal. The next element is the silicon carbide rhodium-coated spherical collımating mirror. The mirror is designed to vertically collimate the radiation in order to better match the inherent SR vertical divergence to the acceptance of the monochromator, but it has little effect on the horizontal beam divergence. An incidence angle of $3 \mathrm{mrad}$ was chosen to give a high-energy cutoff matched to the K-edge absorption of the rhodium coating at $23 \mathrm{keV}$. The collimating mirror is planned as a future addition to the beamlne optics. The next element is the white-beam monitor, which operates by detecting the photoelectrons emitted from a tungsten mesh ( $90 \%$ transmittance). In combination with the white-beam slit, this monitor can be used to detect position shifts of the electron beam in 


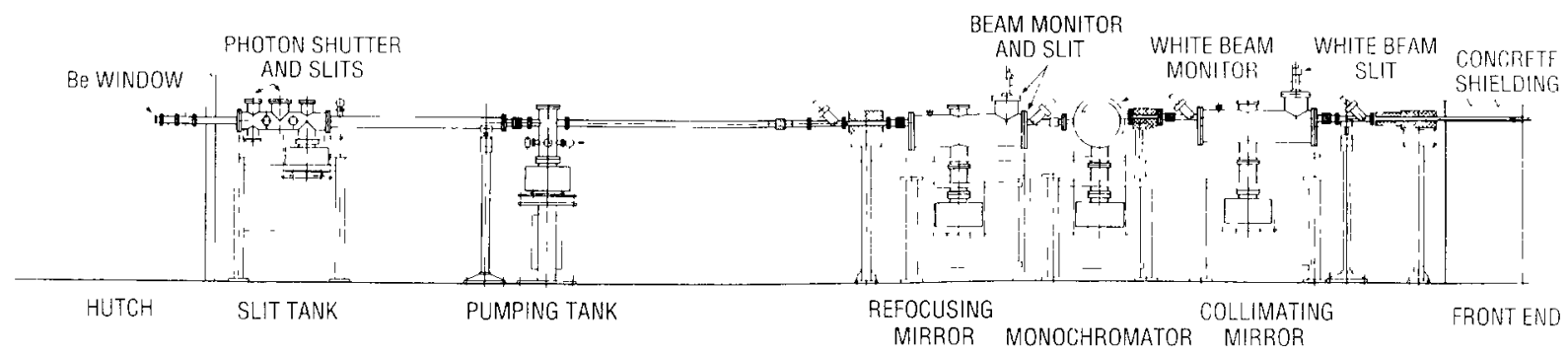

Fig. 1. Layout of the key components of the X-19A beamline at the NSLS.

the storage ring. The monitor is also used by the monochromator feedback electronics. Downstream of this monitor is the NSLS boomerang-type double flat crystal monochromator [1,2]. After leaving the monochromator, the diffracted X-ray beam from the crystals is sampled by the channel electronic multiplier (CEM) monitor. Following the CEM monitor is a cylindrical aluminum refocusing mirror, which is plated with electroless nickel and overcoated with rhodium. This mirror is designed to focus the beam at the sample to a diameter of about $1 \mathrm{~mm}$. The incidence angle of the refocusing mirror is $3 \mathrm{mrad}$, matching the collimating mirror. The focusing mirror can be dropped out of the beam path for unfocused operation. The next elements are the vertical and horizontal hutch slits located just upstream of the hutch. These slits can also be used to improve the energy resolution and to limit the size of the beam to match the sample size. The following element is the photon shutter used to stop radiation from going into the hutch. Use of the photon shutter allows other upstream elements to remain illuminated, thus avoiding thermal transients and thereby improving the performance stability of the beamline. The beamline is maintained at ultrahigh vacuum up to the 10-mil-thick beryllium window located inside the hutch. The beamline is controlled by a MicroVAX II computer with a CAMAC interface running the MicroVMS operating system. The $\mathrm{X}$-ray absorption data collection software at X-19A was written by Engbretson [3].

\section{Beamline characterization and future improvements}

From an experimenter's point of view, the principal concerns for beamline performance are: tunability, available photon fluxes, energy resolution, polarization, and harmonic contamination. The performance of the $\mathrm{X}-19 \mathrm{~A}$ beamline is largely determined by the choice of monochromator crystals. The monochromator has two presettable Bragg angle ranges: $8^{\circ}$ to $15.5^{\circ}$ and $14.97^{\circ}$ to $69.14^{\circ}$. With $\mathrm{Si}(111)$ crystals, these angular ranges correspond to two energy ranges of $2.12<E<7.93 \mathrm{keV}$ and $7.4<E<13.53 \mathrm{keV}$ respectively. The energy range can be extended to $22.10 \mathrm{keV}$ with Si (220) crystals.
Since the monochromator is installed in a UHV condition, even lower-energy operation will be possible in the future. The soft-X-ray capability of this beamline could be extended by using large $d$-spacing crystals [4] such as InSb (111), YB $\mathrm{B}_{66}$, and beryl.

Available photon fluxes were calculated using a ray tracing program [5] for Si (111) crystals in an unfocused mode with a $4 \times 40 \mathrm{~mm}^{2}$ beam size at a ring current of $100 \mathrm{~mA}$. The calculated photon fluxes of $10^{10}, 5 \times 10^{11}$, and $10^{11}$ photons $/ \mathrm{s}$ are predicted for photon energies at $2.5,5$ and $10 \mathrm{keV}$ respectively. The actual photon fluxes were estımated using an ion chamber with $\mathrm{N}_{2}$ gas over the energy range of $2.5-7.9 \mathrm{keV}$. The observed photon fluxes are found to be an order of magnitude smaller than the predicted fluxes. A more careful investigation of this is currently under way, and further optimization of the various beamline components will be conducted. However, the current fluxes of about $10^{9}-10^{10}$ photons $/ \mathrm{s}$ are still adequate to obtain experimental data with a good signal-to-noise ratio in many cases.

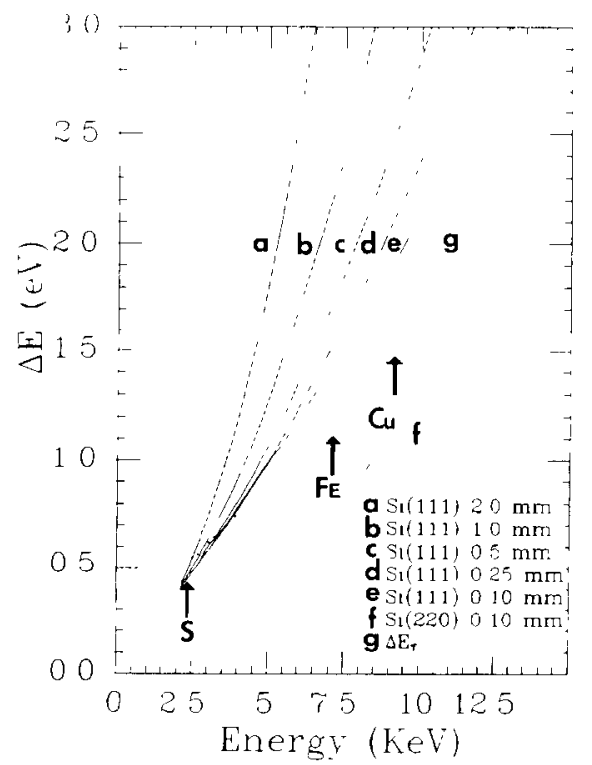

Fig. 2. Expected energy resolution of the monochromator for $S_{1}$ (111) and $S_{1}(220)$ crystals with different slit sittings. 
At the X-19A beamline, two factors contribute to the total energy resolution: the intrinsic reflection width of the monochromator crystals and the angular aperture allowed by the slit systems and the 0.25 -mm-high source point at the NSLS. The total energy resolution of the monochromator as a function of energy is shown in fig. 2. As one can see, the energy resolution of $\mathrm{Si}$ (111) crystals with a proper slit is well matched to the intrinsic lifetıme broadening [6], $\Delta E_{\tau}$, of absorption edges up to $5 \mathrm{keV}$. In order to improve the monochromator resolution for energies above $5 \mathrm{keV}$, one can use $\mathrm{Si}$ (220) crystals. The degree of polarization of the beam was calculated by the same ray tracing procedure [5]. We found that the beam was $98 \%$ polarized in the horizontal direction and $2 \%$ in the vertical direction at $10 \mathrm{keV}$. The calculation indicated that the degree of polarization should not vary significantly as a function of energy. In general, the harmonic contamination can be reduced by detuning the monochromator. At lower energies, the 10-mil Be window functions as a high-pass filter [7], i.e., it absorbs most of the fundamental and transmits most of the higher orders. Thus detuning is needed so that the angular misalignment between the monochromator crystals exceeds the rocking curve width of the harmonic lines. In lower-energy operation, the photon intensity is usually reduced by about $80-90 \%$ by the detuning procedure. Significant improvement in harmonic rejection will be achieved using a grazing incidence mirror. Gains in 1ntensity by a factor of 3-4 may be expected from this.

\section{Improvements of the monochromator system}

At the X-19A beamline, the monochromator consists of two flat parallel single crystals constrained by a mechanical linkage system. The geometry of the monochromator employs the "right-angle" linkage which was first developed by Golouschenko et al. [1]. With mechanical bearings replacing air bearings, a new UHV compatıble version of this type of monochromator was reported by Cowan et al. [2]. Three characteristics of this monochromator are worth mentioning. 1) Ideally, the output beam from the monochromator is fixed in both position and angle as the energy is changed. The major advantage of the fixed exit geometry is to eliminate the need to track the beam motion with postmonochromator optics such as a downstream mirror or a table on which the sample and detectors are placed. 2) The monochromator can be operated in UHV. The system can be operated in a windowless beamlıne, without degrading the storage ring vacuum. 3) Only one motor is needed to produce an energy scan.

There are two practical concerns for this monochromator system: maintaining relative angular alınment durıng a scan and maintainıng UHV compatıbility. In this type of non-dispersive double-crystal monochromator, the two crystals are arranged to diffract sequentially at the same Bragg angle. The monochromator throughput is a convolution of the rocking curves of the individual crystals. Thus, misalignment of the relative angle from parallelism between the two crystals will lead to a significant loss of intensity. Ideally, it is desirable to maintain parallelism of the crystals. (In other words, the crystals would simply remain parallel without additional effort.) Unfortunately, errors induced by the mechanical slides tend to increase with the length of travel, and thus for large angular scans it is difficult to maintain sufficient angular alignment between the two crystals. Over the angular range from $5^{\circ}$ to $70^{\circ}$, the angular error in crystal parallelism is about 30 arc sec due to the tolerances of the system. Furthermore, two general problems associated with the properties of crystals and the nature of SR are anticipated, which can strongly affect the alignment of the crystals. First, the monochromator crystals not only reflect the fundamental, but also higher orders. In order to filter out unwanted reflections, higher harmonics can be suppressed by adjusting the pair of crystals to be slightly non-parallel. For example, with Si (111) crystals at $10 \mathrm{keV}$, the intensity of the third harmonic can be reduced by $10^{3}$ with an angular deviation of about 4 arc sec. Although the current monochromator has a water-cooled front crystal, the problem of considerable heat loading on the first crystal $\left(\sim 50^{\circ} \mathrm{C}\right)$ in the white beam still exists. This can change the lattice constant of one of the two crystals relative to the other due to different thermal expansion. Based on the above considerations, contınual angular correction for this type of monochromator is essential to proper beamline operation.

In practice, piezoelectric transducers (PZTs) with an analog feedback control circuit are used to apply a correction angle or fine tunıng to the second crystal. After the Bragg angle of the first crystal is set to the desired energy, the PZT mechanism gives the second crystal two additional angular degrees of freedom. These control the relative angle between the two crystals and the alignment of the diffracting planes perpendicular to the reflection plane. The former is the most critical adjustment. This is achieved by operating on the side of the rocking curve of the second crystal as a function of the relative angular alignment over a small range, i.e., looking at the reflected intensity from the monochromator as a function of the voltage applied to the second crystal PZT. The analog feedback system used to control the PZT bias is modeled on the system described by Krolzig et al. [8]. It monitors the efficiency of the monochromator by forming the ratio of two signals, one proportional to the white beam flux entering the monochromator, and a second proportional to the monochromatic output flux. In our case, the signals measured by the white-beam monıtor and the ion chamber in the 
hutch are used as input signals for the feedback system. The PZT bias is varied by a dc feedback circuit to hold the ratio of these signals equal to a reference signal. This approach easily permits detuning for harmonic rejection, and works well at maintaining a constant output flux from the monochromator during an XAS scan.

One characteristic of this monochromator is that it operates in UHV. To achieve UHV compatibility, the internal bearings of the monochromator could not be lubricated with oils or greases. However, we had difficulty in operating the monochromator for more than about one month without encountering a bearing failure. This problem now appears to be resolved, after we conducted separate UHV tests of commercial stainless steel bearings with and without UHV-compatible dry lubricants. In our case, the fit of the bearings in the monochromator mechanısm seemed crucial; the bearıng must fit onto its shaft and into its housing using only light finger pressure. Also, the dry lubricant must be very thin, to prevent removal of all radial free play in the unloaded bearing.

\section{Initial X-ray absorption results}

The capabilities of this beamline for X-ray absorption studies are illustrated by the spectra in figs. 3 and 4. The energy resolution of the monochromator was tested by measuring the $\mathrm{Mn} \mathrm{K}$-edge of $\mathrm{KMnO}_{4}$ as shown in fig. 3 using $\mathrm{Si}(111)$ crystals with a $0.5-\mathrm{mm}$ slit. We estimate the experimental monochromator resolution (fig. 3) to be about $1.5 \mathrm{eV}$, as predicted in fig. 2 . Higher-resolution edge spectra could be obtained by

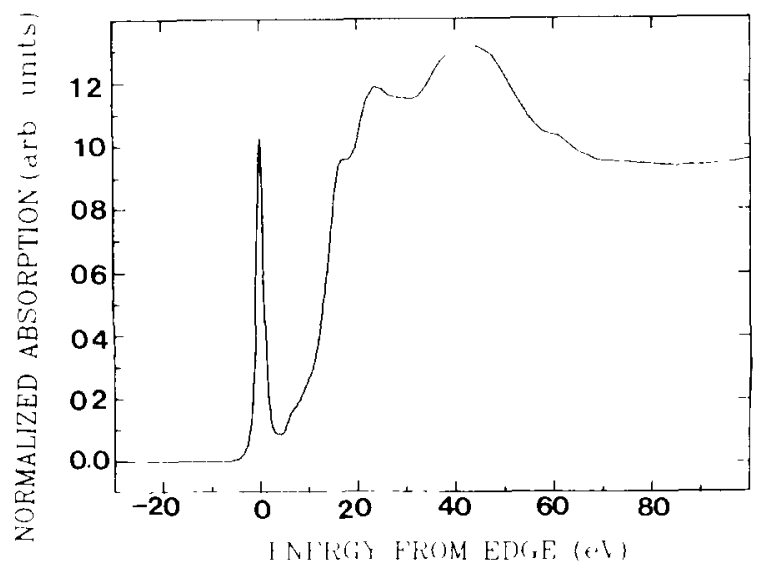

Fig. 3 Near-edge spectrum of $\mathrm{Mn} \mathrm{K}$-edge of $\mathrm{KMnO}_{4}$, measured with $S_{1}$ (111) crystals and slit of $0.5 \mathrm{~mm}$. From this measurement we estımate that the resolutıon of the monochromator is about $1.5 \mathrm{eV}$ as expected.

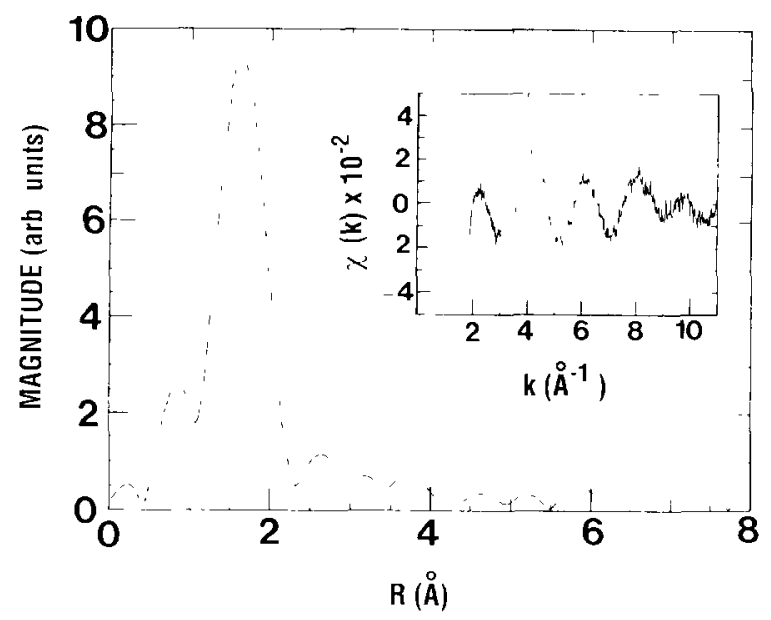

Fig. 4. Fourter transform of $\mathrm{S}_{8}\left[10\right.$ at $\%$ in $\left.\mathrm{BN}_{3}\right]$. The $k^{2}$ transform was taken over a $k$-space region of $2-10 \AA^{-1}$. The inset shows the quality of EXAFS data.

using crystals with higher index diffraction planes with an optimum slit setting.

An EXAFS spectrum and its corresponding $k^{2}$ Fourier transform of the $S$ K-edge of $S_{8}[10$ at.\% in $\mathrm{BN}_{3}$ l are shown in fig. 4. The EXAFS spectrum was measured using $\mathrm{Si}$ (111) crystals. The monochromator was detuned by about $80 \%$. The beam size was approximately $4 \times 20 \mathrm{~mm}^{2}$, and the intensity was estimated to be about $10^{8}$ photons/s. The data was collected using an ion chamber fluorescence detector [9] with $1 \mathrm{~s}$ acquisition tıme per data point. Other good signal-to-noise ratio sulfur edge spectra for highly dilute systems are also published in these Proceedings [7]. During this run, the NSLS was operated at an energy of $2.5 \mathrm{GeV}$ and electron current from 200 to $90 \mathrm{~mA}$. Due to the brightness of the NSLS X-ray ring and the existing experimental setup of the X-19A beamline, we feel that high quality fluorescence EXAFS spectra for low- $Z$ elements ( $\mathrm{P}, \mathrm{S}$, and $\mathrm{Cl}$ ) can be obtaned in reasonable time for lower-concentration samples of about 0.01 at. $\%$.

\section{Further applications}

This beamline was designed for hıgh resolution XAS measurements over a wide energy range. Initial results demonstrate that, with a further improvement of the monochromator, this goal has been accomplished. A unique feature of this beamline is its UHV compatibility. In its current configuration, the single thick Be window absorbs about $95 \%$ of the photons at $2.5 \mathrm{keV}$. Despite this limitation, we obtained a good signal-tonoise ratio for dilute samples comparable to that reported using the Stanford Synchrotron Radiation 
Laboratory 54-pole undulator [10]. In the future, the $\mathrm{Be}$ window will be either removed (for UHV-compatible samples) or replaced with a thin diamond window to permit even lower-energy studies. This will open a new spectroscopic window for studies of samples of biological, chemical, and materials science interest in the lowenergy $\mathrm{X}$-ray range (1-3 $\mathrm{keV})$.

\section{Acknowledgement}

We wish to thank Steve Heald and Daniel Fischer for helpful discussions.

\section{References}

[1] J A Golovchenko, R.A Levesque and P.L. Cowan, Rev. Sc1. Instr 52 (1981) 509.

[2] P.L. Cowan. J.B Hastıngs, T Jach and J.P. Kurkland, Nucl. Instr. and Meth. 208 (1983) 349.

[3] M. Engbretson, unpublished.

[4] J. Wong et al., Nucl. Instr and Meth. 195 (1982) 133.

[5] S.M. Heald and J.B. Hastıngs. Nucl. Instr. and Meth. 187 (1981) 553

[6] M.H. Chen and B. Crasemann. Phys. Rev. A21 (1980) 436, and references therein.

[7] D.A Fischer and C Y. Yang, these Proceedings (6th Nat. Conf. on Synchrotron Radiation Instrumentation. Berkeley, CA, USA, 1989) Nucl. Instr and Meth. A291 (1990) 123.

[8] A. Krolzıg, G. Materlık, M. Swars and J. Zegenhagen, Nucl. Instr and Meth. 219 (1984) 430.

[9] F.E. Lytle et al., Nucl Instr. and Meth. 226 (1984) 542

[10] B. Hedman et al.. Nucl. Instr and Meth. A246 (1986) 797. 